\title{
A Big Moment for Exciton
}

\section{Tunable excitons in bilayer graphene}

Authors: Long Ju, Lei Wang, Ting Cao, Takashi Taniguchi, Kenji Watanabe, Steven G. Louie, Farhan Rana, Jiwoong Park, James Hone, Feng Wang, Paul L. McEuen, Science 358, 1314 (2018).

\section{Recommended with a Commentary by Liang Fu, MIT}

An exciton is an electrically neutral quasiparticle in insulating states, comprised of an electron and a hole bound to each other by Coulomb attraction. The motion of the electron orbiting around the hole results in quantized energy levels of the exciton, similar to those in the hydrogen atom. These internal states of the exciton are responsible for optical absorption peaks at discrete energies below the quasiparticle band gap.

The highlighted paper reports the observation of excitons in bilayer graphene, with tunable exciton energy spectra and giant magnetic splitting. In high-quality bilayer graphene devices under infrared illumination, two sharp absorption peaks with narrow line widths are found. The first peak, isolated from the rest of the absorption spectrum, is identified as the lowest-energy exciton state, the $1 s$ state. Two prominent features are observed. First, under an applied electric field between the two graphene layers, the exciton state moves to higher energy because the displacement field increases the single-particle band gap. The optical absorption frequency can thus be electrically tuned from mid-infrared to terahertz. This functionality may enable tunable infrared detectors based on bilayer graphene.

The most interesting discovery of this work in my opinion is the splitting of the exciton $1 s$ peak in a magnetic field perpendicular to the sample plane. This energy splitting increases linearly with the magnetic field, corresponding to an effective g-factor of about 20 . This means that the exciton $1 s$ state is doubly degenerate at zero magnetic field, and the two degenerate states carry out-of-plane magnetic moments in opposite directions, 10 times larger than the spin magnetic moment of a free electron. This large exciton magnetic moment cannot come from electron's spin, as the optically bright exciton is spin-singlet (note also that spin-orbit coupling is negligible in graphene). As for possible orbital contributions, conduction and valence bands come from carbon $p_{z}$ orbitals. Also, the $1 s$ exciton state has zero orbital angular momentum associated with the motion of electron-hole center of mass. Then, where does such large magnetic moment come from?

The two-fold degeneracy of exciton states in bilayer graphene result from the presence of two valleys located at opposite corners of the Brillouin zone $\pm K$. At a given valley say $+K$, Bloch wavefunction of the conduction band reside mostly on $A$ sublattice of the top layer, while the valence band on $B$ sublattice of the bottom layer, as illustrated in Fig.1. These two types of wavefunctions transform differently under the three-fold rotation around, say, an $A$ site: the $A$ sublattice wavefunction is invariant, whereas the $B$ sublattice wavefunction 
picks up a nontrivial phase factor $e^{i 2 \pi / 3}$ due to the Bloch phase modulation at the lattice scale. Therefore the conduction and valence bands at $+K$ carry different angular momenta $L_{c}=0$ and $L_{v}=+1$ respectively. Because of this Bloch-wave angular momentum (see also Ref.[1]), the $1 s$ exciton state from $+K$ valley in bilayer graphene carries a total angular momentum $L=-1$, while its time-reversed partner from $-K$ valley carries $L=+1$. Then by symmetry, such excitons should carry valley-contrasting magnetic moment.

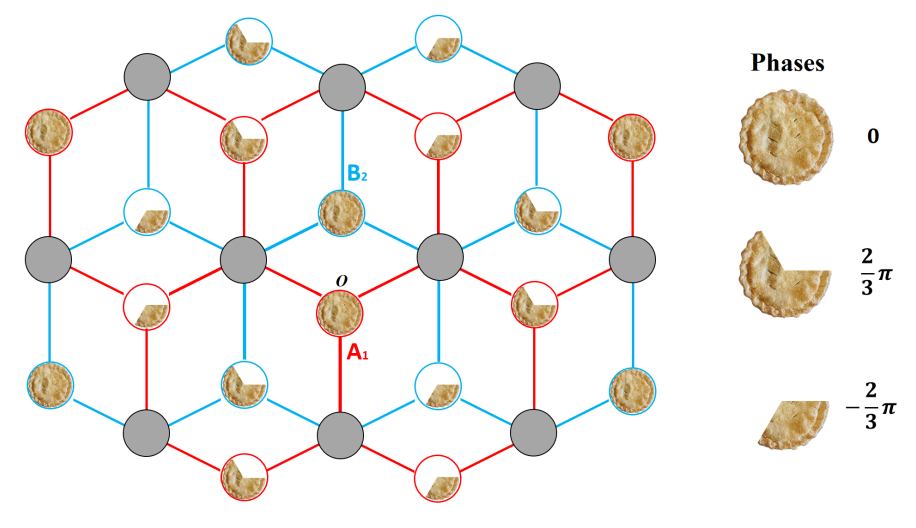

Figure 1: Phase distribution of conduction and valence band wavefunctions of gapped bilayer graphene at $+K$ point. Here conduction band wavefunction is mainly at $A_{1}$ sites (red) of layer 1 and valence band wavefunction is mainly at $B_{2}$ (blue) sites of layer 2 . The rotation center $O$ is chosen as one $A_{1}$ site.

It remains an interesting open question why the moment is so large. As the authors pointed out, this is likely due to the Dirac-like band structure of bilayer graphene, with nontrivial pseudospin texture and Berry curvature in momentum space. Theory predicts that a single-particle wavepacket at energy close to the gap edge is self-rotating [2]. One may then expect the exciton acquires its magnetic moment from these spinning electron and hole. A full theory for the orbital magnetic moment of excitons remains to be developed.

Another direction for future research is to study the exciton spectra in bilayer graphene at high magnetic field, where single-particle Landau levels start to appear. Recent capacitance measurements show that as a function of displacement field, bilayer graphene at charge neutrality exhibits various quantum Hall states with distinctive spin, valley and orbital polarizations [3]. Measurement of exciton spectra may shed new insight into the nature of these quantum Hall states.

\section{References}

[1] C. H. Park, S. G. Louie, Nano Lett. 10, 426431 (2010).

[2] Di Xiao, Wang Yao, and Qian Niu, Phys. Rev. Lett. 99, 236809 (2007).

[3] B.M. Hunt, J.I.A. Li, A.A. Zibrov, L. Wang, T. Taniguchi, K. Watanabe, J. Hone, C. R. Dean, M. Zaletel, R.C. Ashoori, A. F. Young, Nature Communications 8, 948 (2017). 